\title{
Ergonomic evaluation of a wheelchair transportation securement system
}

\author{
Madiha Ahmed, $\mathrm{MS}^{\mathrm{a}}$, Naira Campbell-Kyureghyan, $\mathrm{PhD}^{\mathrm{a}},{ }^{*}$, Karen Frost, $\mathrm{PhD}^{\mathrm{b}}$, Gina Bertocci, $\mathrm{PhD}^{\mathrm{b}}$ \\ andustrial and Manufacturing Engineering Department, P.O. Box 784, University of Wisconsin-Milwaukee, \\ Milwaukee, WI, 53201, USA \\ ${ }^{\mathrm{b}}$ Mechanical Engineering Department, University of Louisville, 500 S Preston St., Louisville, KY, 40202, USA
}

\begin{abstract}
The Americans with Disabilities Act (ADA) specifies guidelines covering the securement system and environment for wheeled mobility device (WhMD) passengers on the public bus system in the United States, referred to as the wheelchair tiedown and occupant restraint system (WTORS). The misuse or disuse of the WTORS system can be a source of injury for WhMD passengers riding the buses. The purpose of this study was to quantify the risks posed to the bus driver while performing the WTORS procedure using traditional ergonomic analysis methods. Four bus drivers completed the WTORS procedure for a representative passenger seated in three different WhMDs: manual wheelchair (MWC), scooter (SCTR), and power wheelchair (PWC). Potential work-related risks were identified using the four most applicable ergonomic assessment tools: PLIBEL, RULA, REBA, and iLMM. Task evaluation results revealed high levels of risk to be present to drivers during the WTORS procedure. The securement station space design and equipment layout were identified as contributing factors forcing drivers to adopt awkward postures while performing the WTORS task. These risk factors are known contributors to injury and the drivers could opt to improperly secure the passengers to avoid that risk.
\end{abstract}

Keywords: Wheelchair transportation safety, ergonomics, task evaluation, disability

\section{Introduction}

The Americans with Disabilities Act (ADA) of 1990 enacted guidelines to protect individuals with disabilities from experiencing discrimination in public settings, including public transportation. Persons seated in wheeled mobility devices (WhMD) use their devices as motor vehicle seats when riding public transportation. Public transit buses in the United States have designated securement stations, as seen in Figure 1, installed with 4 wheelchair tiedowns (TDs) and an occupant restraint system (ORS) consisting of a lap and shoulder belt for use by WhMD passengers. The wheelchair tiedown and occupant restraint system is collectively referred to as "WTORS".
Frost et. al [8] observed WTORS practices at a metropolitan public transit provider and found that the majority of WhMDs (76\%) were unsecured (no wheelchair tiedowns were used) during transport. Furthermore, misuse of the lap belt to secure the WhMD was observed frequently ( $44 \%$ of cases), and consisted of bus drivers using the lap belt to attempt to secure the wheelchair by wrapping the lap belt around the wheelchair seatback. Improper or no securement has been cited as contributing to injuries in $35 \%$ of motor vehicle injuries involving WhMD passengers over a five year period of time [13, 14].

Proper use of the WTORS consists of attaching the 4 TDs to welded sections of the WhMD frame ( 2 in front and 2 in the rear) and restraining the occupant using both a lap and shoulder belt. WhMD passengers

\footnotetext{
${ }^{*}$ Corresponding author: Email: campbeln@uwm.edu - Mail: P.O.Box 784, Milwaukee, WI, 53201- Phone: $4142293403-$ Fax: 4142296814
} 
are dependent on bus drivers to secure their WhMD since the equipment is located beyond their reach. Proper WTORS practice by bus drivers may reduce WhMD passenger related injuries, but current efforts to improve wheelchair transportation safety have focused on the securement hardware, device design and the WMD passenger's perspectives. To the authors' knowledge, no studies have been performed investigating the WTORS task with an ergonomics perspective which would incorporate the risks present to the bus driver (the user of the WTORS system) in the evaluation. Thus, the purpose of this study is to identify the potential work-related risk factors using an ergonomic evaluation and analysis of the WTORS procedure.

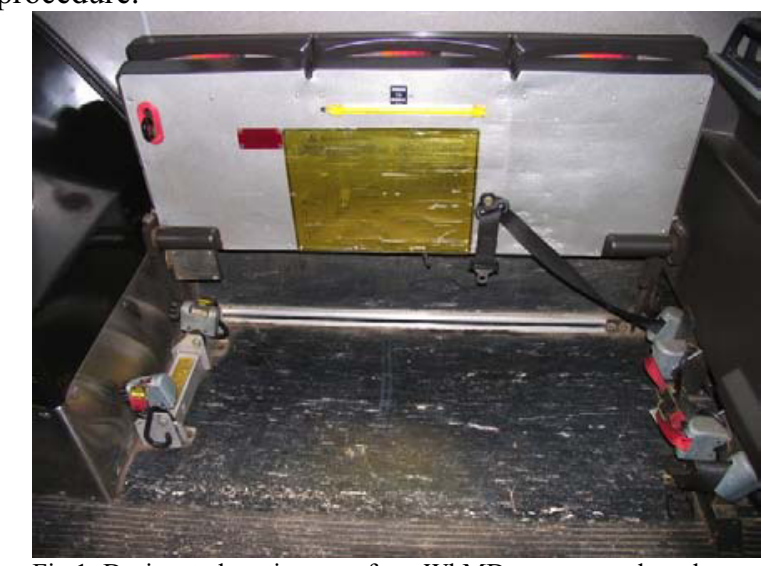

Fig 1: Designated seating area for a WhMD passenger aboard a US bus with the securement equipment (4 tie-downs). Not shown is the occupant restraint.

\section{Methods}

The study was conducted at a metropolitan public transit provider in accordance with a research protocol approved by the Institutional Review Board. The experiment was conducted in an operating Gillig model 2008 low-floor, large accessible transit vehicle while parked at the bus depot. Bus drivers properly applied WTORS to three different WhMDs [manual wheelchair (MWC), scooter (SCTR), and power wheelchair (PWC)] while a single individual sat in each for the duration of the experiment. The order of WhMDs was randomly assigned for each bus driver. All bus drivers had been previously trained by the transit agency in the application of WTORS and did not receive additional instructions from the researchers regarding the securement procedure, except to emulate realistic operating conditions and secure the substitute individual as if other passengers were aboard the bus. Drivers chose the order of equipment securement (TD or ORS) and were videotaped during the experiment for task evaluation.

\subsection{Subjects}

Four, full-time bus drivers employed at the transit agency volunteered to participate in this study. On average, the drivers were $49(+12)$ yrs old, weighed $96(+45) \mathrm{kg}$, and stood $1.76(+.13) \mathrm{m}$ tall. Two bus drivers were male and all were right handed.

\subsection{Task Evaluation}

Potential work-related risks were identified using the four most applicable ergonomic tools: PLIBEL [9]; Rapid Upper Limb Analysis (RULA) [10]; the Rapid Entire Body Assessment (REBA) [12]; and the industrial lumbar motion monitor (iLMM) [15]. The first three tools captured the presence of risk factors specifically pertaining to the task layout, task procedures, and drivers' body postures during securement and are known to be reliable. PLIBEL and RULA have been validated $[9,10]$. The iLMM provided a direct biomechanical measurement of the driver's spinal motions throughout the task and has been validated to accurately identify those jobs which may lead to a low back injury [17]. Evaluations of the WTORS task with the first three tools were completed after three evaluators trained in ergonomic job analysis scored the tasks and consensus was determined for all tasks.

PLIBEL is a 17-item checklist that indicates the presence of work-related risk factors linked to musculoskeletal injuries/symptoms in five body regions (neck/shoulder/upper back, elbows/forearms/hands, knees/hips, feet, and low back) [9]. RULA [10] and REBA [12] are more specific ergonomic tools used to evaluate the postural risks to the worker. RULA questions target the upper body while REBA questions are concerned with the entire body. The final scores correlate to varying action levels regarding the task analyzed. In RULA, scores range between 1 (no action necessary) and 7 (action must be taken immediately). The final score is a composite score of the two subgroup scores (groups A and B). Group A reflects the items pertaining to the arm and hands while the Group B relates to the neck, trunk, and leg analysis. In REBA, the scores range between 1 (low risk and no action needed) and 15 (very high risk and action must be taken immediately). The final score is also a composite score of two subgroup scores (Group A relates to the neck, trunk, and legs while Group B relates to the arms and wrists). Subjects were fitted with the iLMM "to monitor the trunk motion's characteristics" [15] throughout the trials. The iLMM risk model determines the probability of any job entering a high risk membership group (i.e. potentially leading to a low back injury). 
For evaluation purposes, the WTORS task was divided into 2 subtasks: 1) application of tiedowns (TDs) to secure the WhMD and 2) application of the ORS (lap and shoulder belts) to restrain the passenger. The WTORS task was assumed to be a no load situation. Other assumptions made during task evaluation were specific to each tool. For PLIBEL, items pertaining to the load, task duration, foot pedal, and seated work design were excluded from evaluation. For RULA and REBA assumptions included no muscle use, lowest load/force build-up situation, and task duration being less than a minute. More assumptions specific to REBA included good coupling and the task being performed less than four times.

PLIBEL scores are separated into body regions: upper (upper back and above) and lower (lower back to feet). The resulting percentage scores is a proportion of the items relating to each body region over the total number of items applicable to that region and then averaged over the four subjects. RULA and REBA final scores (averaged over the four subjects) are presented as well as the different group scores for comparison. Overall iLMM risk model results are presented.

WhMD design and size have been shown to affect task performance $[3,6]$ and may potentially pose different risks to the driver, therefore average task scores are presented by subtask and WhMD type. A repeated measures design was used to evaluate differences in scores based on WhMD and to minimize the effects of subject variability on the resulting task analysis. All statistical analysis was conducted using Microsoft Excel 2007 version for Windows. An alpha level of $\mathrm{p}<0.05$ was used to determine significance. Marginally significant differences were identified for $p$-values between .05 and .1. The magnitude of risk differences between pairs of devices will be discussed as well as noting any significance of device type and evaluated risk.

\section{Results}

Ergonomic analysis from all four tools verifies the existence of risk factors in the WTORS task. Figure 2 presents final results for the 4 tools according to subtask (TD/ORS) and WhMD type. Table 1 depicts the average differences in magnitude between the identified risk and paired devices for each tool.

Average PLIBEL scores (Fig. 2a) ranged between $30(+10) \%$ and $57(+2) \%$ depending on device type and subtask. Overall, more risks were identified when applying the TDs than the OR across all devices, but there was a larger variation in how the subjects secured the OR versus the TDs. The PWC was different from the other two devices. Significant differences between the MWC and SCTR existed for only the upper body when securing the TDs ( $p$-value = .028). A higher amount of risks were identified for the upper body in both subtasks than for the lower body. Securing the SCTR generated the highest amount of risks to the upper body in both subtasks ( $57 \%$ for TDs and $51 \%$ for OR). The same descending order of risks identified by device was observed for both subtasks: SCTR generated the highest amount of risk, followed by the PWC and then the MWC. In the lower body however, the order of risks identified by device were opposite for the two subtasks. For the TD subtask, the highest amount of risk in the lower body was identified when securing the PWC, followed by the SCTR, and then the MWC. The exact opposite was true for the OR subtask. Small difference magnitudes between paired devices and the identified risks to the upper body were seen; whereas larger differences were observed with the identified lower body risks seen in Table 1.

RULA scores (Figure 2b) identified both subtasks as high risk (scores above 6). Application of the WTORS to the MWC generated the largest RULA score for both subtasks. Applying TDs was associated with higher scores and was subject to less variation as compared to applying the OR. Marginally significant differences were observed when applying TDs to the MWC and PWC (p-value $=0.057)$. Group A scores (arms/hands) generated a larger range of action levels (can wait for action to need action now) than Group B scores (need action soon to need action now).

Between WhMD types, there was minimal difference for both group scores for each subtask (Table 1).

Average final REBA scores (Figure 2c) indicates the WTORS task to generally be of at least high risk. Applying TDs resulted in higher scores for all WhMD types compared to applying the ORS. All of the final scores in the TD subtask were associated with a very high risk level and required action to be taken immediately (scores greater than 11). In the OR subtask, the PWC device was associated with the highest risk among all three WhMDs. Marginally significant differences were observed between the MWC and PWC for the TD (p-value $=.057)$ and OR (p-value =.057). Group A heavily influenced REBA's final scores. For the TD subtask, Group A scores indicated the final score would be either a high or a very high risk task. For the OR subtask, group A scores indicated a lower risk range for the final score (medium to high risk). Group B scores had a larger spread for resulting risk in the final scores (low to high risk), especially for the TD subtask. There was little difference in the magnitudes of average Group A scores, but score differences were larger for the Group 
B scores (Table 1) when applying the ORS (average scores range between .25-.63).

Results of the iLMM risk model (Figure 2d) indicate the likelihood of the WTORS task entering high-risk group membership based on subtask and WhMD. Probabilities are based on five variables: lift rate, average twisting velocity, maximum moment, maximum sagittal flexion, and maximum lateral velocity [8]. In the WTORS task the lift rate and maximum moment did not contribute to the final result. The TD task is more likely to become high risk task than the OR subtask. Of all devices, securing the MWC is more likely to become a high risk task (average score of $32+4 \%$ ) than the other two devices. The probabilities for the OR subtask ranged between
$22+2 \%$ and $24+7 \%$ ). All three devices were different for both subtasks (no significant differences observed from subtask scores).

The ergonomic analyses revealed the presence of work-related risk factors when performing the WTORS task. All four ergonomic tools identified securing the TDs to be the riskier subtask and a large variability in how subjects secured the OR. There was not a general consensus on which device generated higher risk for the WTORS task between the four tools. The MWC had higher risk from more tools for the TD subtask. MWC and PWC were statistically found to be the same device according to three of the tools (PLIBEL, RULA, and REBA) when securing the TDs.

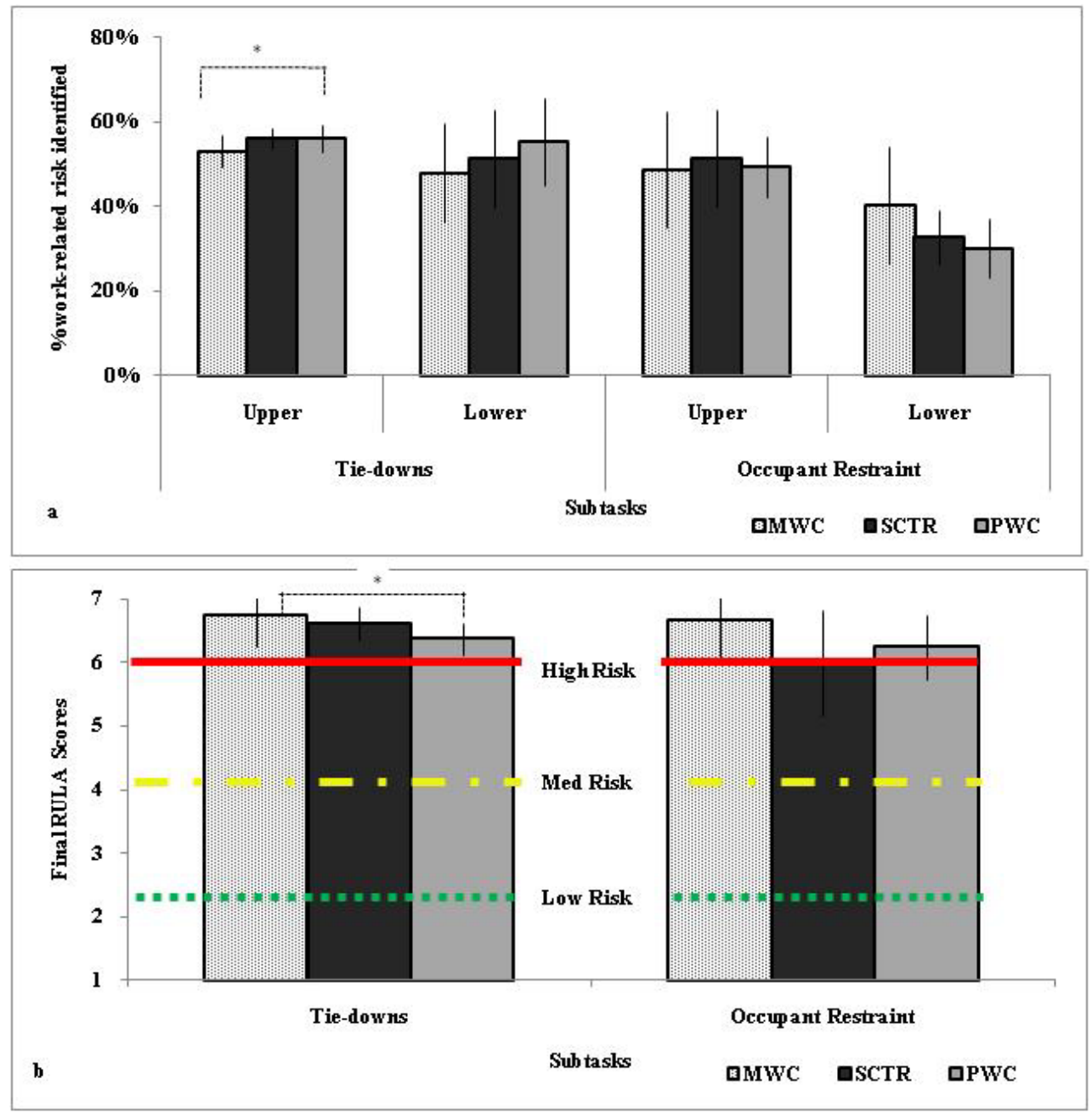



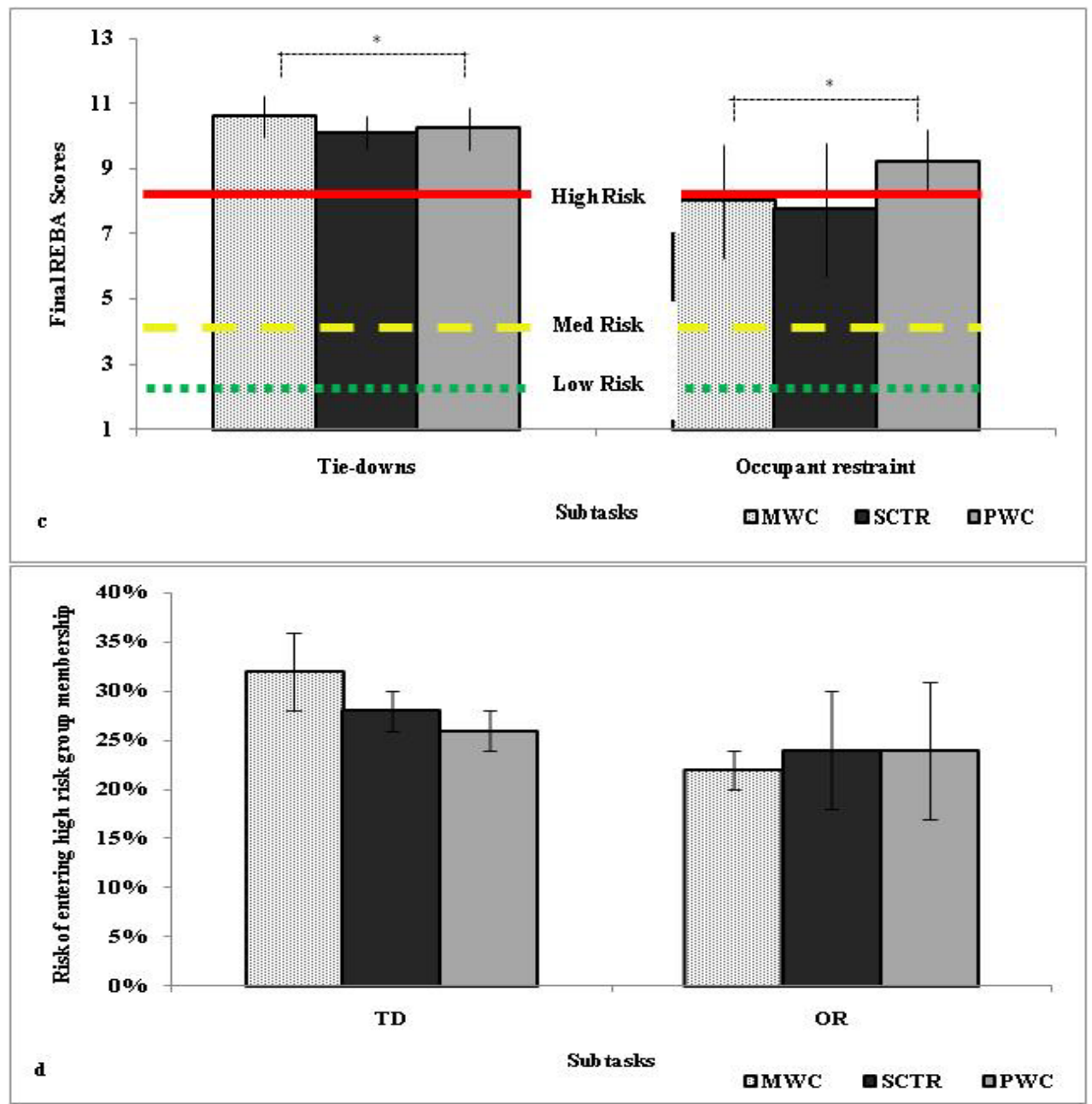

Fig 2: Overall average ergonomic task analysis results shown by device type for each subtask (a) PLIBEL (b) RULA (c) REBA and (d) iLMM. PLIBEL scores are shown for the upper and lower body. Final RULA and REBA scores and corresponding risk levels are shown. The probability of the WTORS task entering a high risk membership group according to the iLMM risk model is shown. *Pair of devices are significant; $\mathrm{p}<.05$ (paired t-test)

Table 1

Magnitude differences in average risk scores for paired devices. $\left.{ }^{*}\right)$ Pair of devices that are significant; $\mathrm{p}<.05$ (paired t-test)

\begin{tabular}{|cc|ccc|ccc|}
\cline { 2 - 7 } \multicolumn{1}{c|}{} & \multicolumn{3}{c|}{ TIE-DOWNS } & \multicolumn{3}{c|}{ OCCUPANT RESTRAINT } \\
\hline \multirow{2}{*}{ TOOL } & MWC-SCTR & MWC-PWC & SCTR-PWC & MWC-SCTR & MWC-PWC & SCTR-PWC \\
\hline \multirow{2}{*}{ PLIBEL } & Upper Body & $6 \pm 3 \% *$ & $6 \pm 4 \%$ & $0 \pm 2 \%$ & $7 \pm 17 \%$ & $7 \pm 32 \%$ & $0 \pm 24 \%$ \\
& Lower Body & $8 \pm 21 \%$ & $19 \pm 29 \%$ & $9 \pm 13 \%$ & $10 \pm 38 \%$ & $15 \pm 43 \%$ & $8 \pm 15 \%$ \\
\hline \multirow{2}{*}{ RELA } & Group A & $0.0 \pm .2$ & $.1 \pm .2$ & $.09 \pm .1$ & $.02 \pm .2$ & $.15 \pm .1$ & $.13 \pm .3$ \\
& Group B & $.08 \pm .1$ & $.09 \pm .1$ & $.02 \pm .0$ & $.11 \pm .1$ & $.04 \pm .2$ & $.03 \pm .3$ \\
& Group A & $.04 \pm .1$ & $0.0 \pm .1$ & $.05 \pm .1$ & $.06 \pm 1$ & $.06 \pm .1$ & $.06 \pm .2$ \\
\hline & Group B & $.12 \pm .1$ & $.22 \pm .1$ & $.1 \pm .1$ & $.43 \pm .9$ & $.63 \pm .8$ & $.25 \pm .4$ \\
\hline
\end{tabular}




\section{Discussion}

Improving the WTORS procedure usage is one way of reducing injuries sustained by WhMD safety passengers. This study is the first to apply an ergonomic perspective and to incorporate the bus operator's role in the WTORS task. The purpose of this study was to evaluate the WTORS task and identify work-related risk factors that could contribute to the driver's risk of injury. WTORS procedure observations revealed several factors that could potentially contribute to work-related injury: including space constraints, bending, twisting, reaching, and diminished visual capacity and the observations were verified with the task analysis results. All four tools identified high levels of risk factors present while securing a WhMD passenger for both subtasks (TDs and OR) and for all three WhMDs (MWC, SCTR, and PWC). Multiple tools were used in task evaluation to account for the limitations of each tool. General consensus among all four evaluation tools indicates that the WTORS task is actually risky and the results are not a product of subjective evaluation. Applying the TDs was found to be of higher risk than applying the OR for all four analysis tools. This general consensus could be attributed to the station design and equipment layout. The designated securement station aboard buses meets the minimum dimension requirements set by the $\mathrm{ADA}$ guidelines. The TDs are installed into the floor of the bus and drivers must flex forward, bend, reach, and twist to retrieve the equipment, locate the correct securement sites on the device, and attach the TDs all while attempting to not invade the seated WhMD passenger's personal space. More risks were identified for the upper body when securing the TDs from PLIBEL and the final scores from RULA and REBA were heavily influenced by the large scores for the neck, trunk, and legs. When securing the TDs, trunk flexion and awkward neck posture contributed to the large group score from RULA. The large group score in REBA is due to the trunk flexion and knee angle when accessing the equipment.

The most influential factor in the iLMM model results was identified as the maximum sagittal flexion. The second most influential factor was the lateral bending due to the subjects trying to secure the TDs in the confined space. Studies have linked these awkward postures and manual material handling tasks to a worker's risk of injury $[1,2,4,5,7,11,16]$. Consequences of such injuries range from an acute pain to long-term overexertion injuries. Individually, any one of these motions can be a contributor to injury. A combination of these motions (such as observed in the WTORS task), can substantially increase the likelihood of the driver sustaining an injury while securing a WhMD passenger.

The results from all four tools showed less variability in how the subjects secured the TDs than for securing the OR. Since the TDs were out of the comfortable zone of reach, all of the subjects had to access the equipment by bending or reaching. In PLIBEL, there was less variation observed for the risks to the upper body than in risks identified for the lower body. All of the operators bent their trunks and used awkward postures of their neck. Yet, there were a variety of lower body postures assumed when securing the TDs. The subjects stooped, squatted, or kneeled while securing the TDs to the devices and hence the larger variation seen for the lower body.

Risks identified when securing the OR were largely due to the large shoulder abduction angle required to reach the shoulder belt. The operators maintained a more neutral posture as compared to the TDs because the OR equipment is physically located at a higher level than the TDs. Not all of the subjects had both feet planted firmly on the ground which can interfere with the driver's balance. The larger variation observed could be due to equipment location and procedure. In the current configuration, the shoulder belt must be accessed while the WhMD passenger is in the station. The operators must reach over the passenger and device, and invasion of personal space is inevitable. Larger variations could also be due to the operators trying to minimize the amount of personal invasion while securing the passenger.

This study also investigated the effect on the identified risks due to the different devices. Device type did have some influence on identified ergonomic risks, yet Table 1 shows the magnitude of risk between paired devices to be generally less than $20 \%$ (for PLIBEL and ILMM) and between .5-2 points (group scores for RULA and REBA). Risk levels by device are generally in the same range within subjects when securing the TDs, but vary more when securing the OR. This variation could be attributed the various postures taken when accessing and securing the OR, as described above. Each subject secured the OR in a different manner for each of the devices. There was not a general consensus between tools as to which device generated the higher amount of risk. Generally, the MWC and PWC were found to be similar devices when identifying risks. The SCTR has a different structure, material make-up, and dimensions and was not expected to be similar to the other two. However, even though the SCTR was different, the identified risks were the same. The operators still needed to bend, reach, and twist to access the equipment and to attach them to the devices. 


\section{Conclusion}

Guaranteeing safe transit to WhMD passengers aboard buses is required by the ADA. Improper securement has been cited as the top injury producing source for these passengers. Efforts to improve the safety have focused on modifying the devices or equipment aboard the buses. This study was the first to incorporate the bus operator's role in the WTORS process. The task evaluation results show high levels of risks to be present while the drivers secure a WhMD passenger. These risks are known contributors to injury and the drivers could opt to improperly secure the passengers to avoid that risk. Also, the securement station space design and equipment layout force the operator to adopt awkward postures while performing the WTORS task. Future efforts to improve the WhMD passenger safety should incorporate the driver's role in the wheelchair transportation process. Ergonomic analysis reveals the urgent need for a task redesign. Design focus should include the usability of the system.

\section{References}

[1] A. Burdorf, and G. Sorock, Positive and negative evidence of risk factors for back disorders Scandinavian Journal of Work, Environment \& Health, 1997. 23(4) p. 243-256.

[2] A. Mital, A. Pennathur, and A. Kansal, Nonfatal occupational injuries in the United States Part II-back injuries1 International Journal of Industrial Ergonomics, 1999. 25(2) p. 131-150.

[3] C. Foreman, and J. Hardin, The challenges of wheelchair securement: Searching for solutions. Tampa, FL National
Center for Urban Transportation Research-Center for Transit Research, University of Southern Florida, 2001

[4] C. Haslegrave, M. Tracy, and E. Corlett, Force exertion in awkward working postures-strength capability while twisting or working overhead Ergonomics, 1997, 40(12) p. 1335-1356.

[5] D.B. Chaffin, G. Andersson, and B.J. Martin, Occupational biomechanics. 1991, Wiley New York.

[6] G. Shaw, and T. Gillispie, Appropriate protection for wheelchair riders on public transit buses Journal of rehabilitation research and development, 2003.40(4) p.309-320.

[7] K. Granata and S. Wilson, Trunk posture and spinal stability Clinical biomechanics-bristol, 2001. 16 p. 650-659.

[8] K.L. Frost and G. Bertocci, Retrospective review of adverse incidents involving passengers seated in wheeled mobility devices while traveling in large accessible transit vehicles Medical engineering \& physics. 32(3) p. 230-236.

[9] K. Kemmlert, A method assigned for the identification of ergonomic hazards--PLIBEL Applied Ergonomics, 1995. 26(3) p. 199-211.

[10]L. McAtamney and E. Nigel Corlett, RULA: a survey method for the investigation of work-related upper limb disorders Applied Ergonomics, 1993. 24(2) p. 91-99.

[11]L. Punnett, et al., Back disorders and non-neutral trunk postures of automobile assembly workers Scandinavian Journal of Work, Environment \& Health, 1991. 17(5) p. 337.

[12] S. Hignett and L. McAtamney, Rapid entire body assessment (REBA) Applied Ergonomics, 2000. 31(2) p. 201-206.

[13] S. Plan., et al., Wheelchair user injuries and deaths associated with motor vehicle related incidents Transportation Research, 2009.

[14] NHTSA, Research Note: Wheelchair User Injuries and Deaths Associated with Motor Vehicle Related Incidents. 1997.

[15] W.S. Marras, et al., The role of dynamic three-dimensional trunk motion in occupationally-related low back disorders Spine, 1993. 18(5) p. 617-628.

[16] W.S. Marras, et al., Biomechanical risk factors for occupational related low back disorders Ergonomics, 1995. 38(2) p. 377-410.

[17] W.S. Marras, et. al, Prospective validation of a low-back disorder risk model and assessment of ergonomic interventions associated with manual materials handling tasks. Ergonomics, 2000. 43(11) p.1866-1886 